\title{
SOLITONS: NOVEL APPROACH FOR DISPERSION MANAGEMENT
}

\author{
Suruchi Bala ${ }^{1}$, Anu Sheetal ${ }^{2}$ \\ ${ }^{I}$ G.N.D.U, Regional Campus, Gurdaspur, Punjab, India \\ ${ }^{2}$ G.N.D.U, Regional Campus, Gurdaspur, Punjab, India
}

\begin{abstract}
Soliton is a special kind of wave packet that travels distortion less over long distances. It is a pulse able to maintain its shape and width due to compensation of Self Phase Modulation (SPM) process which is a non-linear effect based on refractive index variation and Group Velocity Dispersion (GVD) process which is a linear effect. For long range communication, soliton pulses are very suitable as the pulse width remain constant over the entire transmission distance. It eliminates the need to cope with any type of dispersion. The transmission of light in optical fiber given by Nonlinear Schrödinger equation has been discussed in the paper. The performance of fiber with soliton parameters is compared to that with fiber without soliton parameters using OptiSystem Software.
\end{abstract}

Keywords: Dispersion, GVD, Soliton pulses, SPM

\section{INTRODUCTION}

A solitary wave is a confined wave that ascends from stability between various linear and nonlinear effects. When the optical pulse propagates along the fiber, it experiences GVD and SPM. These effects put limitation on increase in demand of capacity. So, soliton is come into existence.

GVD is a linear effect where refractive index varies with frequency components. When different frequency components travel with different velocity, differential transit time takes place and the pulse gets distorted. Whereas when the nonlinear phase shift of optical signal changes with respect to time, it results in varying frequency called chirping and the phenomena is known as SPM. Positive SPM is caused when refractive index increases with increase in pulse intensity. This alteration in refractive index leads to a phase shift in time domain. The pulse spectrum due to SPM changes, as frequency is the derivative of phase shift in time domain. As a result of combination of the positive GVD and the positive SPM, broadening of the pulse takes place. A pulse with the exact compensation between the negative GVD and the positive SPM, a pulse propagates with no distortion as shown in Fig 1.1.

This astonished, at first, since a change in the time domain and the frequency domain is caused by GVD and SPM respectively. However, a spectrum will retain its shape if any shift in phase is added to a Fourier transform limited pulse in time domain [1]. The pulse propagates with same shape and remains undispersed after travelling long distance, if GVD will cancel this phase shift in the same fiber. Solitons are therefore a remarkable breakthrough in the field of optical fiber communications.
$* * *$

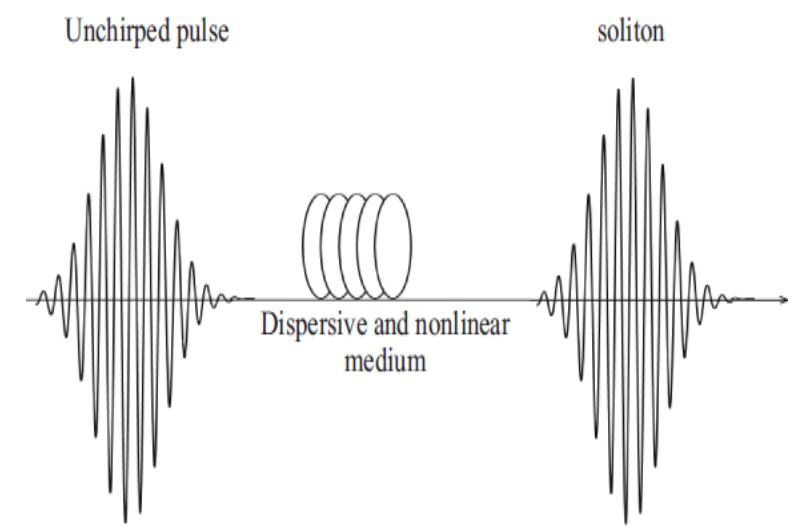

Fig-1.1: Soliton pulse

Solitons are the localized solitary waves that have very distinctive properties: (i) They propagate through the optical fiber at a constant speed by maintaining their shape. (ii) They are enormously stable to the changes produced during propagation especially when collided with small amplitude linear waves. (iii) They remain stable when collision occurs between two soliton pulses. When two soliton pulses collide, they just pass through each other and emerge out by holding their original shape [5].

\section{EVOLUTION OF TEMPORAL SOLITONS}

In order to understand dispersion properties of optical pulses for a single mode fiber, it is important to know how it propagates under influence of dispersive conditions. This has been briefly discussed by the dispersion broadening processes and self-phase-modulated narrowing processes further.

\subsection{Self Phase Modulation}

When the pulse itself induces the phase shift, it results in the variation in frequency of a pulse known as SPM. SPM arises as intensity component of the fiber is linked to the refractive 
index. High refractive index is experienced by the pulse when it propagates along the fiber with high pulse intensity, causes the leading side of pulse to attain a positive refractive index gradient $\left(\frac{d n}{d t}\right)$ and experiences lower refractive index, when it travels with lower pulse intensity, causes the trailing side to attain a negative refractive index gradient $\left(-\frac{d n}{d t}\right)$. Phase shift is induced in the different parts of the pulse due to refractive index variations. This is called frequency edge of the pulse is shown in Fig-2.1. Therefore, main effect of SPM is to spread the pulse by maintaining its temporal shape [6],[7].

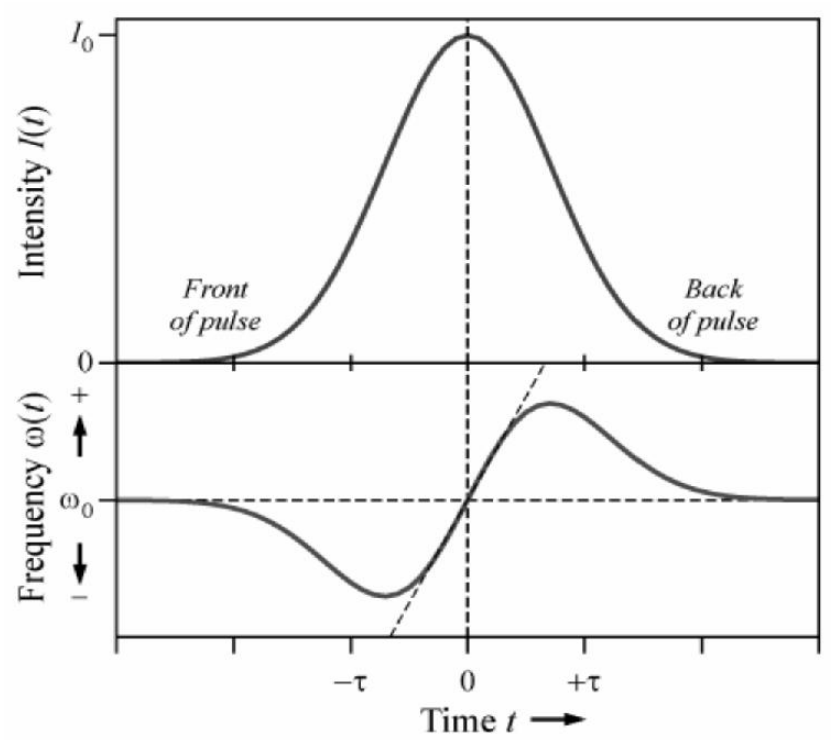

Fig-2.1: Pulse spread due to SPM

For a pulse propagating through the optical fiber of length $\mathrm{L}$ with high pulse intensity $\mathrm{I}$, the phase shift $[\square$ ]isgiven by

$$
\phi=\frac{2 \pi}{\lambda}\left(n_{1}-n_{2} \mathrm{I}\right) L_{e f f}
$$

where $\mathrm{L}_{\text {eff }}$ is the effective length and is given by

$$
L_{e f f}=\frac{1-\exp (-\alpha l)}{\alpha}
$$

$\mathrm{n}_{1}$ is linear R.I, $\mathrm{n}_{2}$ is non-linear R.I and $\alpha$ is attenuation constant.

In equation (1), $\frac{2 \pi}{\lambda} n_{1} L_{e f f}$ is linear phase constant and $\frac{2 \pi}{\lambda} n_{2} \mathrm{I} L_{\text {eff }}$ is non-linear phase constant. This phase change in time domain results in different frequency components.

When the frequency of optical carrier is modulated then new frequency component becomes

$$
\omega^{\prime}=\omega_{0}+\frac{d \phi}{d t}
$$

From equation (1) \& (2)

$$
\frac{d \phi}{d t}=-\frac{2 \pi}{\lambda} L_{e f f} n_{2} \frac{d I}{d t}
$$

Therefore

$$
\omega^{\prime}=\omega_{0}-\frac{2 \pi}{\lambda} L_{e f f} n_{2} \frac{d I}{d t}
$$

At rising edge of pulse $\frac{d I}{d t}>0$

Then

$$
\omega^{\prime}=\omega_{0}-\omega(t)
$$

At trailing edge of pulse $\frac{d I}{d t}<0$

Then

$$
\omega^{\prime}=\omega_{0}+\omega(t)
$$

where $\omega(t)=\frac{2 \pi}{\lambda} L_{e f f} n_{2} \frac{d I}{d t}$

Thus the variations in frequency components occur due to SPM. This variation further leads to spreading of the pulse.

\subsection{Group Velocity Dispersion}

When the optical pulse propagates along the fiber, it experiences a group delay, as pulse consists of different wavelengths, each travelling at a different velocity. This group delay leads to the broadening of the pulse. The pulse spreading causes the adjacent pulses to overlap which further leads to Inter Symbol Interference (ISI) [6],[7]. GVD is also referred as group delay dispersion parameter. The group delay, $I g$ per unit length is given by

$$
\frac{I_{g}}{L}=\frac{1}{V_{g}}=\frac{1}{c} \frac{d \beta}{d k}=\frac{-\lambda^{2}}{2 \pi c} \frac{d \beta}{d \lambda}
$$

where, $L$ is the distance traveled by the pulse, $\square \square$ is the propagation constant along fiber axis, $k$ is the wave propagation constant, $\mathrm{k}=\frac{2 \pi}{\lambda}$ and $v g$ is the group velocity, $v_{g}=c\left(\frac{d \beta}{d b}\right)^{-1}$. The delay difference per unit wavelength can be approximated as, $\frac{d I_{g}}{d \lambda}$ assuming the optical source not to be too wide in spectral width. For spectral width $\square \square$,the total delay difference $\square T$ over distance $L$, can be written as

$$
\delta T=\frac{\delta I_{g}}{d \omega} \delta \omega=\frac{d}{d \omega}\left(\frac{L}{v_{g}}\right) \delta \omega=L\left(d^{2} \beta / d \omega^{2}\right) \delta \omega
$$

where $\omega$ is angular frequency.

The factor $\beta_{2}=d^{2} \beta / d \omega^{2}$, is a GVD parameter which determines quantum of pulse dispersion in time.

The Group Velocity dispersion puts severe limitation on high bit rate information carrying capacity for long distance optical communication systems. A simple solution to this problem is the use of optical soliton pulses that maintain their width over entire transmission distances. Soliton based optical communication systems can be utilized over long 
distances with immeasurable data carrying capacity by using optical enhancers and a speed of the order of $\mathrm{T}$ bit/s can be accomplished if WDM system with optical amplifiers is consolidated in soliton based communication systems.

\section{SOLITON BASED TRANSMISSION}

Soliton is a pulse able to keep its shape and width constant and hence propagate undistorted over long distances and stay unaffected after colliding with other soliton pulses.

-RZ (Return to Zero) refers to digital data transmission in which binary low and high states are represented by 0,1

- NRZ (Not Return to Zero) is a binary code in which 1s are represented by positive voltage.

- Soliton format principally utilizes one soliton to represent ' 1 ' bit.

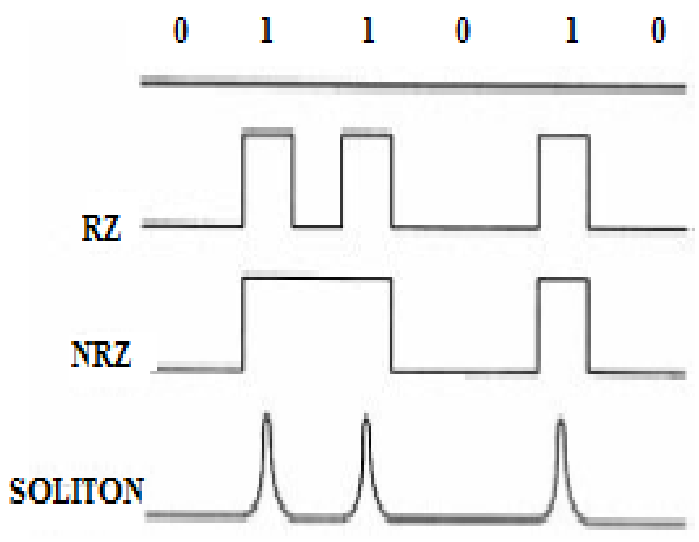

Fig-3.1: RZ, NRZ and SOLITON format

\subsection{Transmission of information}

While utilizing solitons for dispersion management the conventional approach is to represent the entire bit slot with high pulse, but this is possible only in cases where neighboring bit sequence are isolated and the separation is more than FWHM. The same cannot be used for consecutive similar bits hence in order to use soliton RZ format is used for isolation for every slot shown in Fig-3.2.

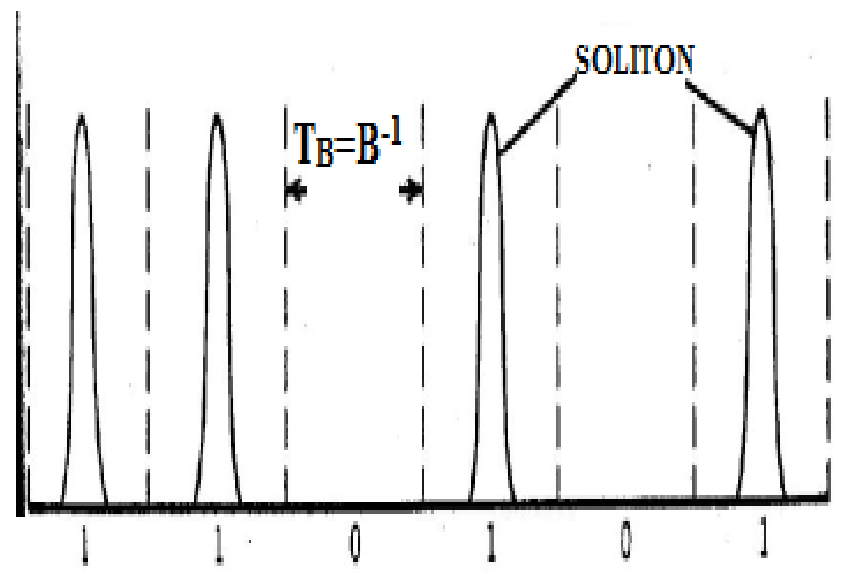

Fig-3.2: Transmitting Soliton pulse using RZ format
The soliton width and Bit rate is related as

$$
\mathrm{B}=1 / \mathrm{T}_{\mathrm{B}}=1 / 2 \mathrm{q}_{0} \mathrm{~T}_{0}
$$

where $T B$ is the Bit duration and between the adjacent solitons, the partition is given by $\mathrm{q}_{0}=\mathrm{T}_{\mathrm{B}} / \mathrm{T}_{0}$. The optical communication system using soliton pulses requires an optical source that generates Pico-second pulses without any frequency chirping. The wavelength region $\sim 1550 \mathrm{~nm}$ is used by the optical source.

\subsection{Soliton in Optical Fiber}

The soliton pulses in optical fibre are present as the result of compensation between the variations induced by fiber dispersion characterized by GVD coefficient $\beta_{2}$ and fiber nonlinearity characterized by SPM coefficient $\omega$. Analytically soliton is a answer of nonlinear Schrodinger equation depicting pulse spreading in optical fiber [5].

The transmission of light in optical fiber is given by nonlinear Schrödinger equation (NLSE). Using standardization parameters for example, the normalized time $T_{0}$, the dispersion length $L_{D}$ and pulse peak power $P_{0}$, the nonlinear Schrödinger equation is given by

$$
i\left(\frac{\partial u}{\partial z}\right)-\frac{s}{2}\left(\frac{\partial^{2} u}{\partial t^{2}}\right)+N^{2}|u|^{2} u+i\left(\frac{\alpha}{2}\right) u=0
$$

where $u(z, t)$ is pulse envelope function, $\mathrm{z}$ is transmission distance along the fiber, soliton order is given by integer $N$ and $\alpha$ is the coefficient of energy where loss in energy is represented by negative values. The value of $s$ is -1 for negative $\beta_{2}$ and +1 for positive $\beta_{2}$ as shown in figure 3.3 and 3.4 .

The fundamental soliton is the pulse with order 1and if order is greater than 1then it is known as higher-order solitons [8]. Order of soliton pulses $(\mathrm{N})$ depends on the balance between dispersion and nonlinearity characteristics and is defined as

$$
N^{2}=\frac{\gamma P_{0}}{\left|\beta_{2}\right|} T_{0}^{2}
$$

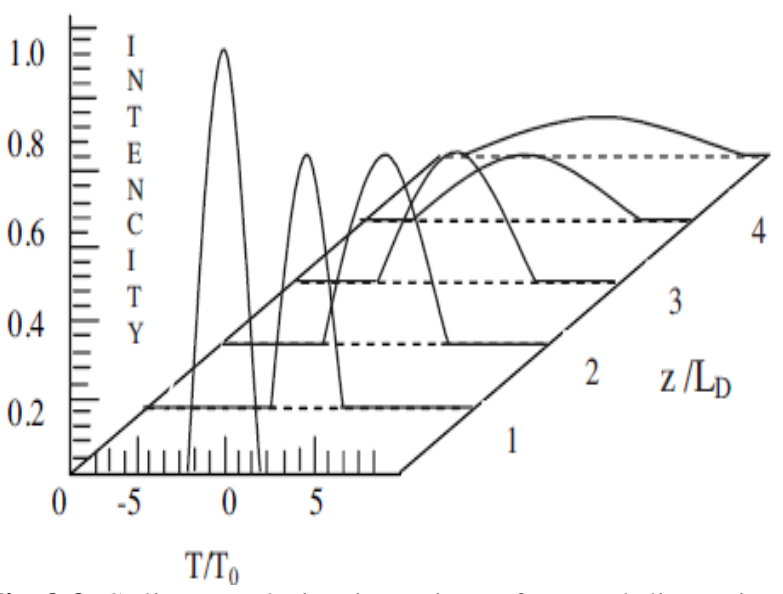

Fig-3.3: Soliton evolution in regime of normal dispersion 


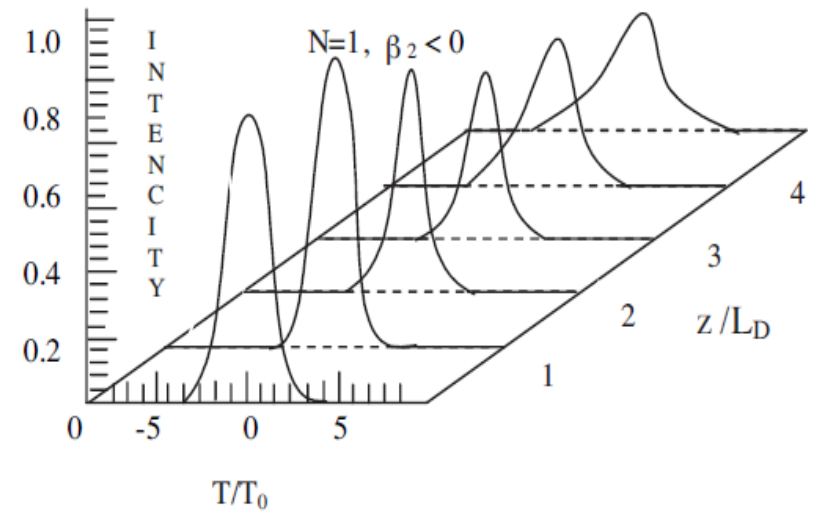

Fig-3.4: Soliton evolution in regime of anomalous dispersion

It is observed that if order of soliton is more than 1 then SPM is high and if order of soliton is less than 1 then GVD increases. At the fundamental order both SPM and GVD cancel each other. So it is efficient to use the fundamental soliton with order 1 as soliton travels undistorted over the entire transmission distance. [5].
The solution for fundamental soliton $(N=1)$ is given by integrating the NLSE and can be written as

$$
u(z, t)=\operatorname{sech}(t) \cdot \exp \left(\frac{i z}{2}\right)
$$

where sech $(t)$ is capacity of hyperbolic secant. Since the shape of the pulse is not changed by the phase term exp $(i z / 2)$, therefore soliton pulses are independent of $z$ and hence remain distortion less in time domain. As a result fundamental soliton pulses are used in optical communication system. Even if the intensity of pulse changes from the stable conditions, no change is noticed in the optical solitons as they are very stable against perturbations.

\section{SIMULATION AND RESULTS}

Experimental setup is shown in Fig-4.1 and Fig-4.6 for the comparative study of using fiber for transmitting information up to $1000 \mathrm{~km}$ with using soliton parameters and without using soliton parameters.

\subsection{Without using Soliton Parameters}

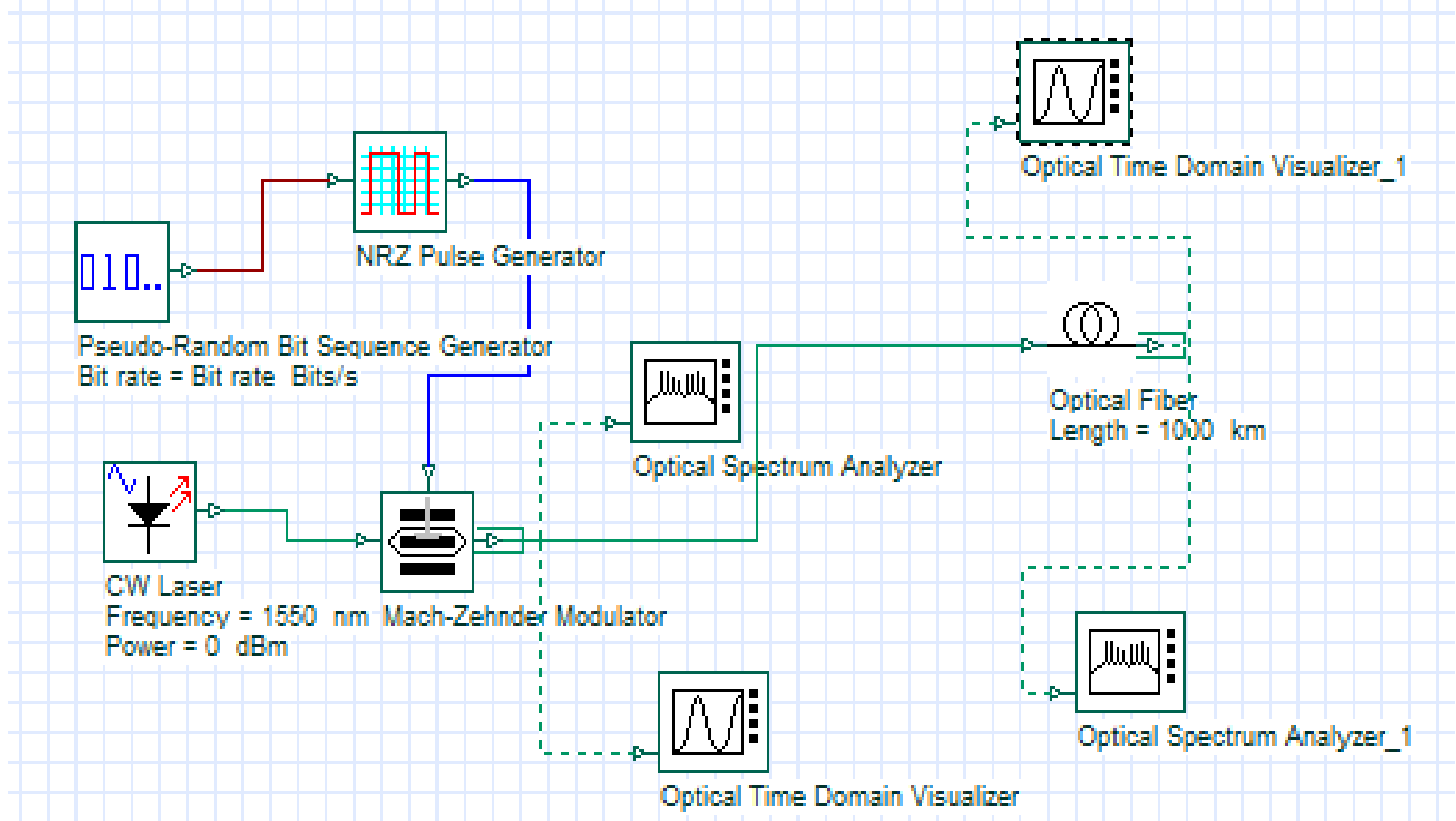

Fig-4.1: System layout 


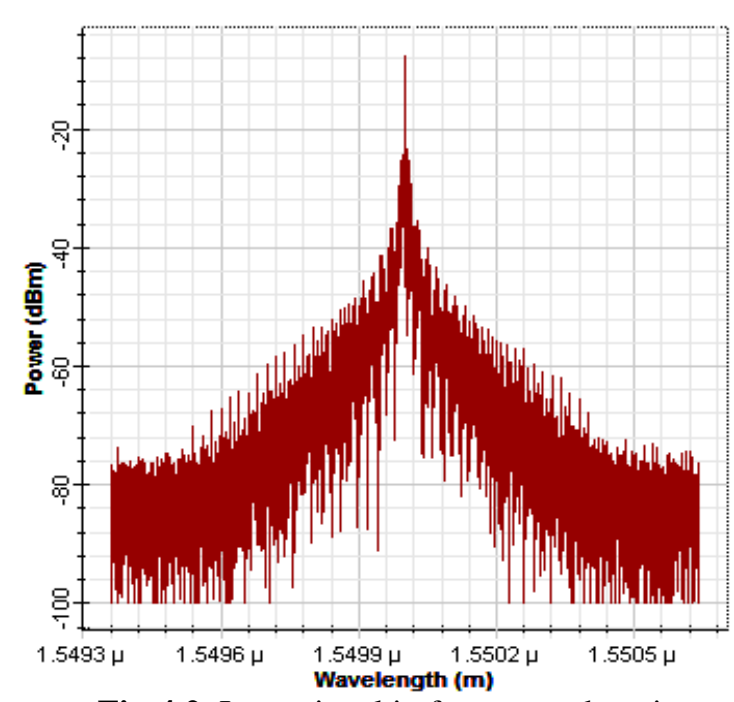

Fig-4.2: Input signal in frequency domain

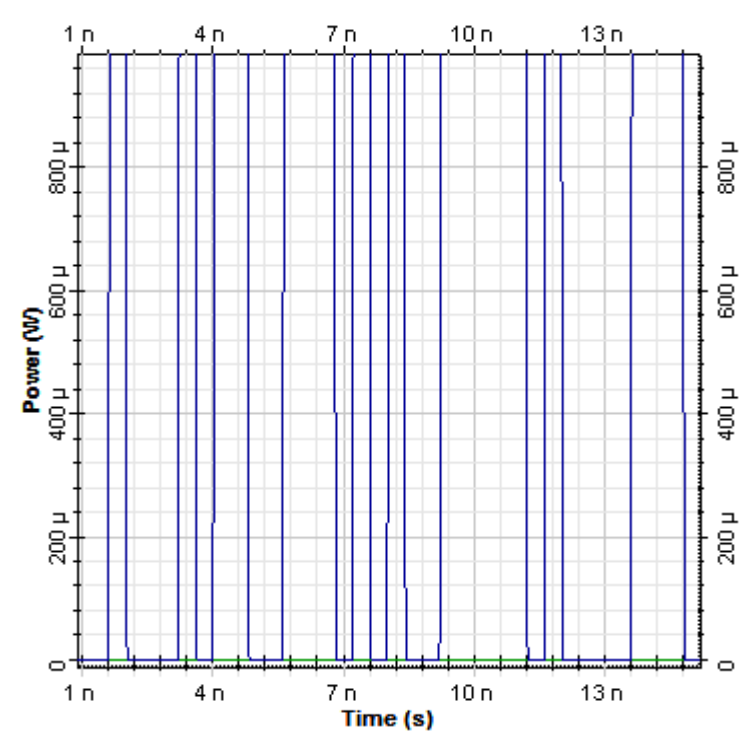

Fig-4.3: Input signal in time domain

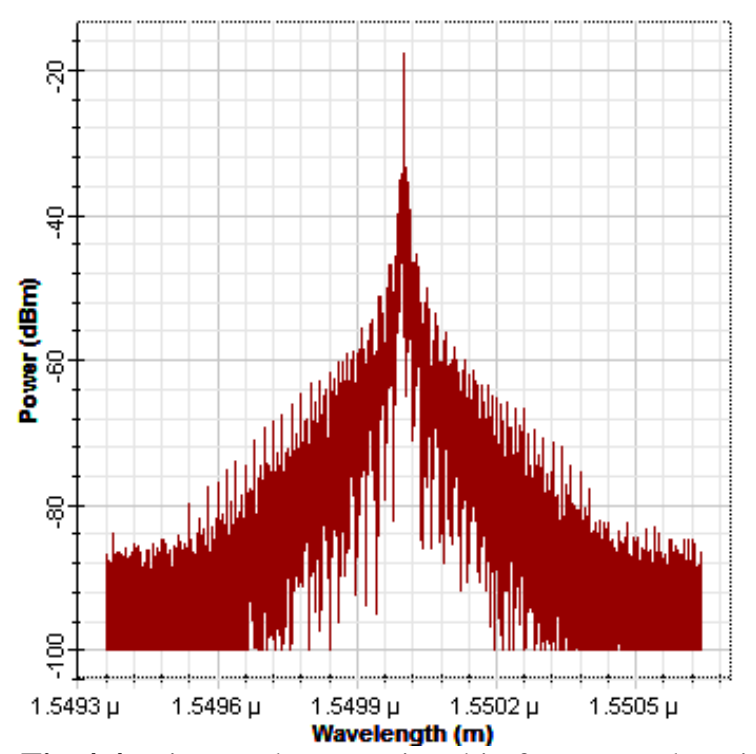

Fig-4.4: Dispersed output signal in frequency domain (without soliton parameters)

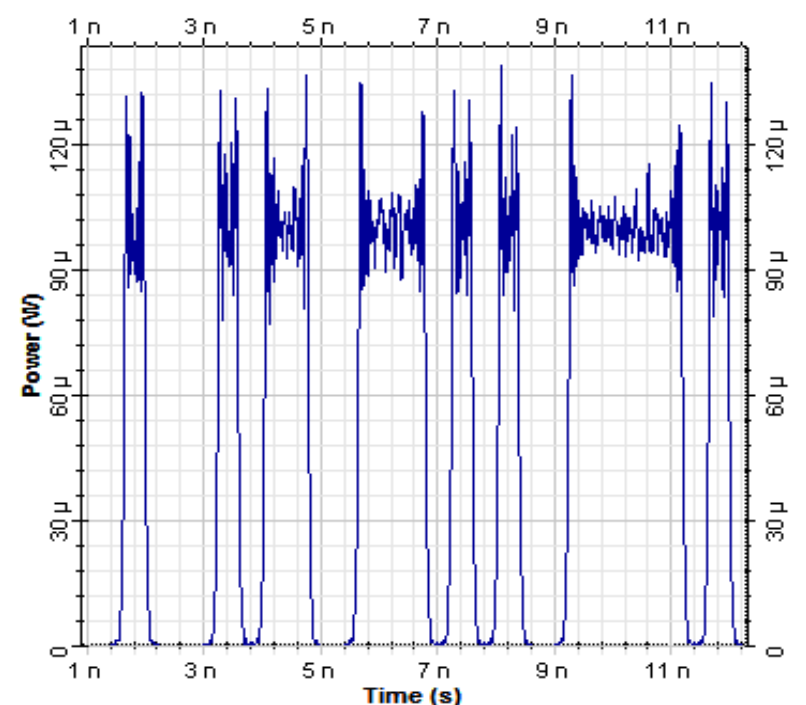

Fig-4.5: Dispersed output signal in time domain (without soliton parameters)

Figure 4.4 and 4.5 shows that the output pulse get dispersed after $1000 \mathrm{~km}$ as GVD and SPM causes the pulse to spread in optical fiber. GVD causes the optical pulse to spread in time domain and degrades the signal over long distance, as pulse contains different wavelengths travelling at different velocities and SPM changes the pulse spectrum as phase shift occurs due to pulse intensity. Both GVD and SPM cause the spreading of pulse. So dispersion management is important in optical communication systems because if dispersion is too high, a group of pulses carrying information will spread in time and merge, resulting in the dispersion of the signal.

One conceivable response to this issue is to utilize Soliton pulses in the anomalous dispersion regime, a form of optical pulse which uses non-linear optical effect to maintain its shape and width. 


\subsection{With using Soliton Parameters}

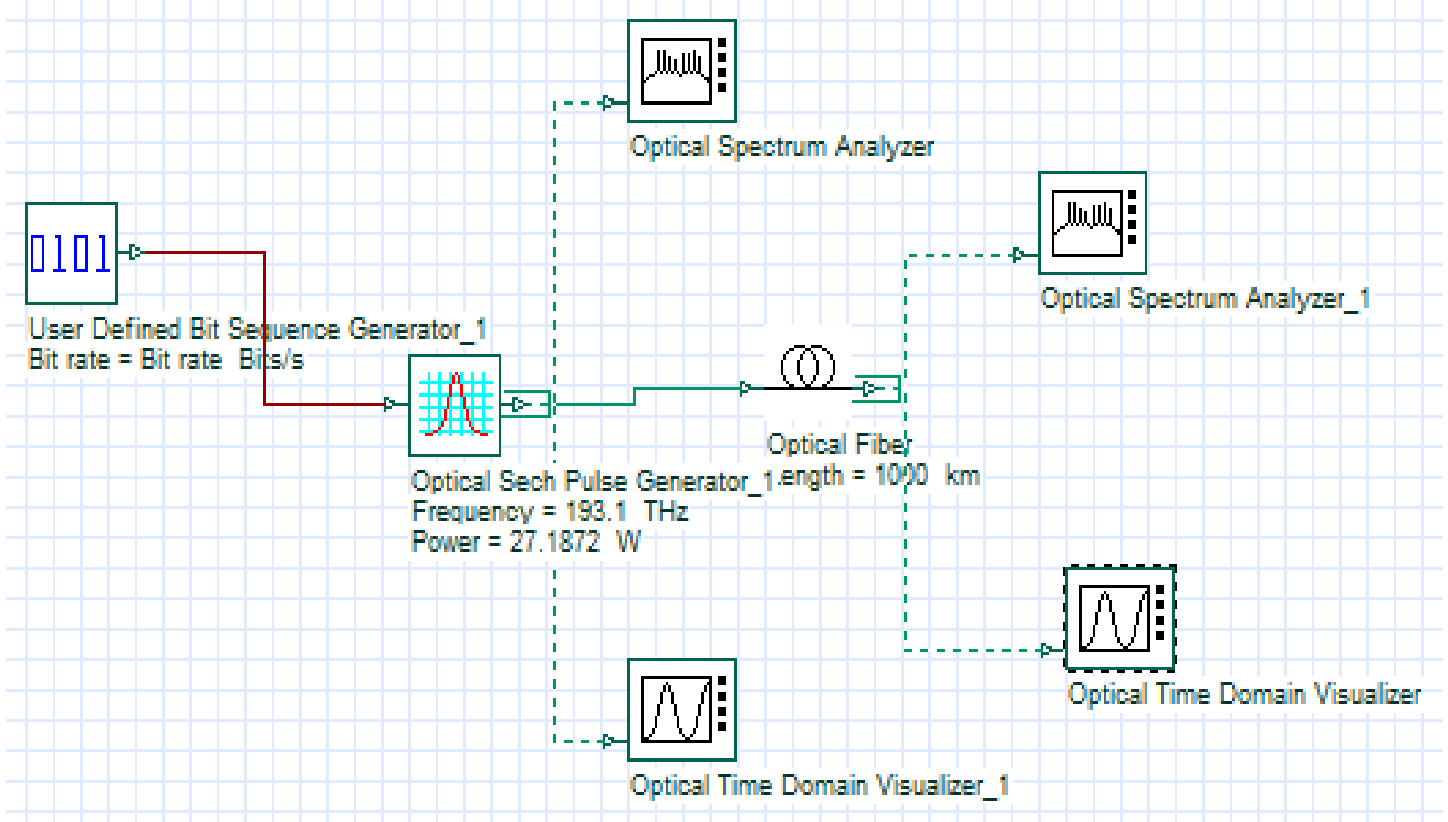

Fig-4.6: System layout

Figure4.6 shows the layout for fundamental soliton $\mathrm{N}=1$.The simulation is done in OptiSystem software. For given $\mathrm{n}_{2}=2.5 \mathrm{e}-20 \mathrm{~m}^{2} / \mathrm{W}, \mathrm{A}_{\mathrm{eff}}=50 \mu \mathrm{m}^{2}$, and $\lambda=1550 \mathrm{~nm}-\gamma=1.5 \mathrm{e}-3$ $1 / \mathrm{m} / \mathrm{W}$. The fiber length is set to $1000 \mathrm{~km}$.

Initial pulse has a sech shape and FWHM pulse width is 33 ps, corresponding to $\mathrm{T}_{0}=15.7$ ps. Pulse power for fundamental soliton is $27.18 \mathrm{~mW}$.

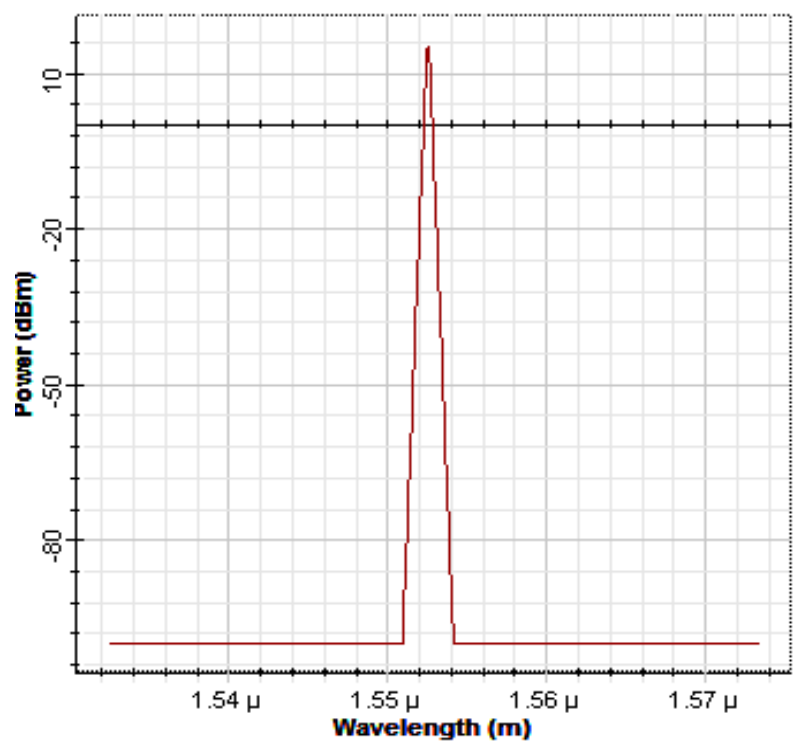

Fig-4.7: Input signal in frequency domain

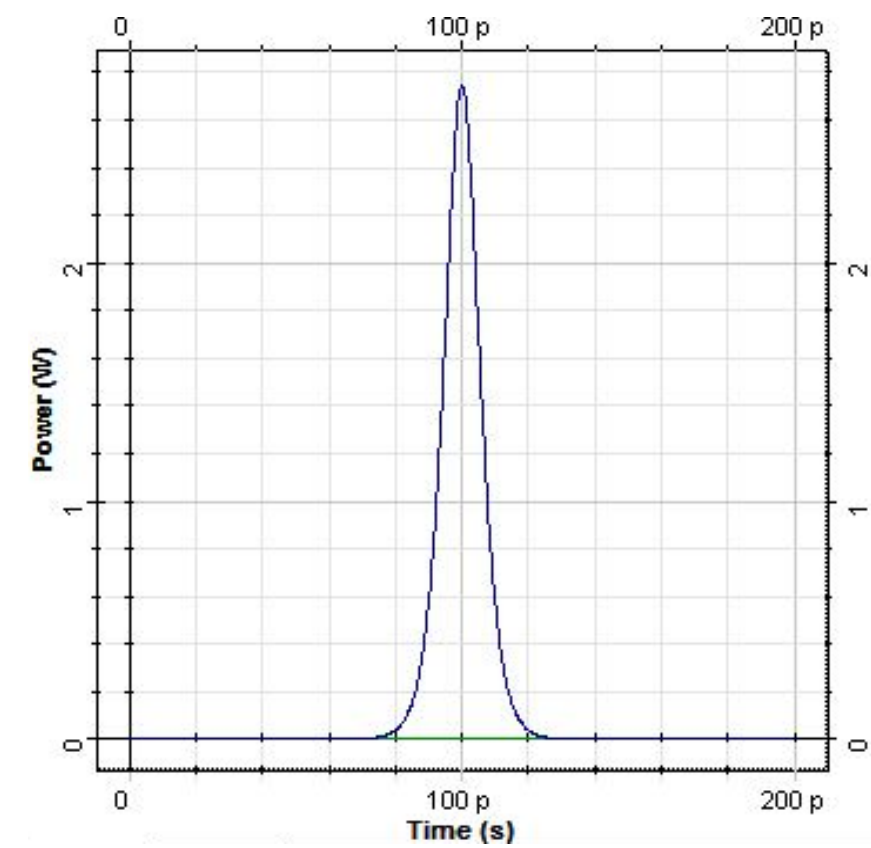

Fig-4.8: Input signal in time domain

Figure 4.9 and 4.10 shows the presence of a fundamental soliton, order $(\mathrm{N}=1)$. The fundamental soliton remains distortion less by both SPM and GVD, since in this case they cancel each other completely. The pulse remains chirp less, due to the exact compensation that occurs between the SPM-induced and GVD-induced frequency modulations. 


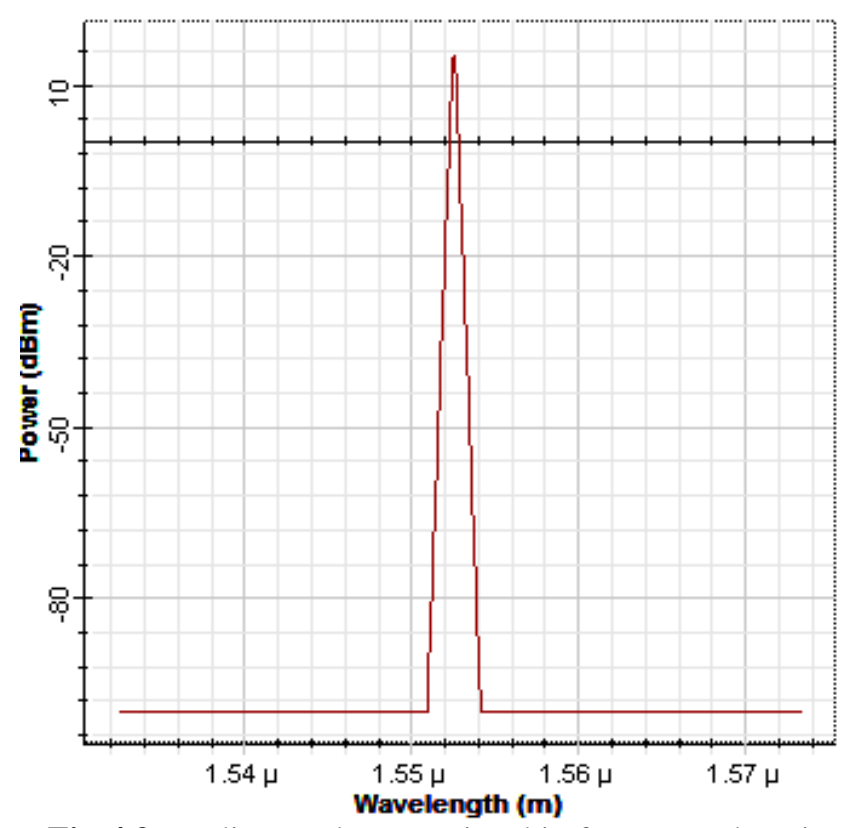

Fig-4.9: Undispersed output signal in frequency domain (with soliton parameters)

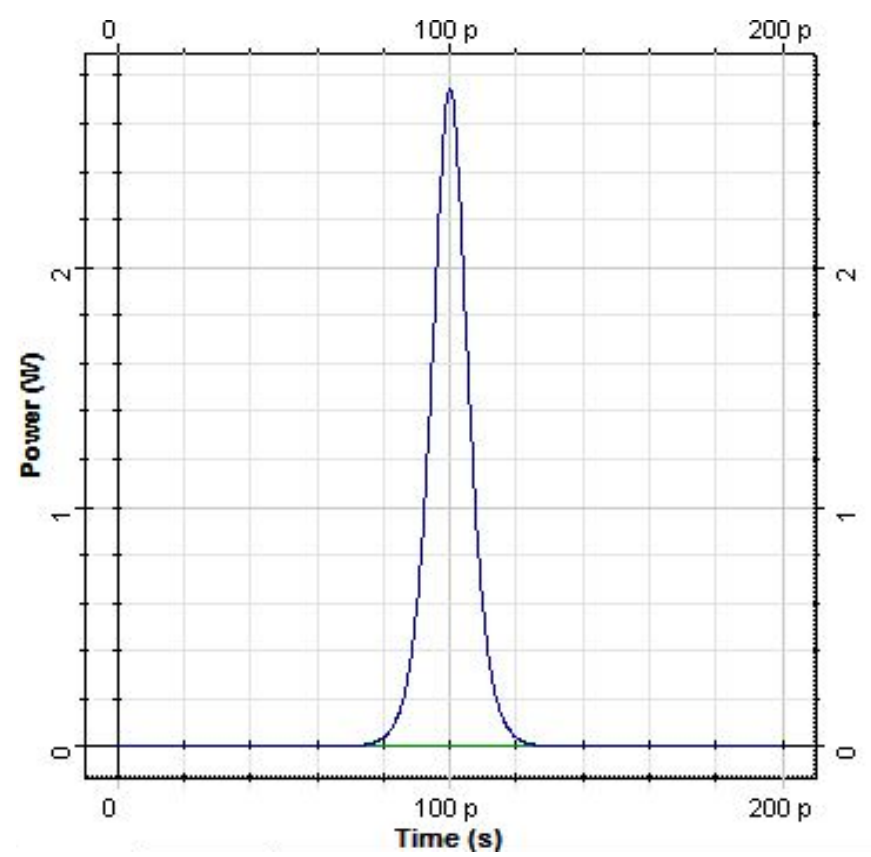

Fig-4.10: Undispersed output signal in time domain (with soliton parameters)

\section{CONCLUSION}

The optical fiber communication systems with soliton pulses are best suitable for long haul communication as they remain undispersed after travelling long distance. This is experimentally shown in the Fig-4.9 and 4.10 that the exact compensation is made between the SPM induced components and GVD induced components, as compare to the system without using soliton parameters shown in Fig4.4 and Fig- 4.5 where the signal get broaden due to various linear and non-linear factors.

\section{REFERENCES}

[1] R. Ganapathy, K. Porsezian, A. Hasegawa, "Soliton interaction under Soliton Dispersion management," IEEE Journal of Quantum electronics, vol.44, no. 04, April 2008.

[2] Samudra Roy and Shyamal K. Bhadra, "Solving Soliton Perturbation Problems by Introducing Rayleigh's Dissipation Function," IEEE Journal of Lightwave Technology, vol.26, no. 14, July15, 2008.

[3] Iao Ioannis Neokosmidis, Thomas Kamalakis, and Thomas Sphicopoulos, "Optical Delay Lines Based on Soliton Propagation in Photonic Crystal Coupled Resonator Optical Waveguides," IEEE Journal of Quantum electronics, vol.43, no. 07, July 2007.

[4] Samuli Kivish, Tommi Hakulinen, "Tunable Raman Soliton Source using Mode-Locked Tm-Ho Fiber Laser," IEEE Photonic Technology Letters, vol.19, no.12, June5,2007.

[5] R.Gangwar, S.P.singh, N.Singh, "Solitons Based Optical Communication," Progress in Electromagnetic Research, PIER 74, pp. 157-166, 2007.

[6] Mehul G. Patel, S.B.Khant, "Soliton Transmission in fiber optics for long distance communication," IJAREEIE, vol.03, no.02, Feb. 2014.

[7] R.L.Sharma, Dr. Ranjit Singh, "Solitons, Its Evolution and Applications in High Speed Optical Communication," International Journal on Emerging Technologies, vol.02, pp.141-145, 2011.

[8] Masataka Nakazawa, Herokazu Kubota, Kazunori Suzuki, "Ultra High Speed Long Distance TDM and WDM Soliton Transmission Technologies," IEEE Journal of selected topics in Quantum electronics, vol.06, no. 02, March/April 2000.

[9] C.Xie, M. Karlsson and P.A. Andrekson, "Soliton Robustness to the Polarization Mode Dispersion in Optical fibers," IEEE Photonic Technology Letters, vol.12, no.07, July,2000.

[10] Akira Hasegawa, "Solitons Based Ultra High Speed Optical Communication," PRAMANA, Journal of Physics, vol.57, pp.1097-1127, Nov. /Dec. 2001.

[11] Norihiko Nishizawa, Ryuji Okamura, Toshi Golo, "Simultaneous Generation Of Wavelength Tunable Two colored Femtosecond Soliton Pulses Using Optical fibers," IEEE Photonic Technology Letters, vol.11, no.04, April 1999.

[12] D.Arbel, Meir Orenstan, "Self Stabilization of Dense Soliton Trains in a Passively Mode-Locked Ring Laser," IEEE Journal of Quantum electronics, vol.35, no. 06, June 1999.

[13] Masao Keto, "Wavelength Tunable Multicolor Raman Soliton Generation Using an Ellipse Polarized Pump Pulse and highly Birefringent Optical fibers," IEEE Journal of Lightwave Technology, vol.24, no. 02, Feb. 2006.

[14] Chongjin Xie, Henrik Sunnerud, "Polarization Mode Dispersion-Induced Outages in Soliton Transmission Systems," IEEE Photonic Technology Letters, vol.13, no.10, Oct. 2001. 
[15] Chongjin Xie, Henrik Sunnerud, "Influences of Polarization Mode Dispersion on Soliton Transmission Systems," IEEE Journal of selected topics in Quantum electronics, vol.08, no. 03, May/June 2002.

[16] Wen-Hua Cao, Kam-Tai Chan, "Generation of Bright and dark Soliton Trains from Continuous-Wave Light Using Cross Phase Modulation in a Nonlinear Optical Loop Mirror ," IEEE Journal of Quantum electronics, vol.37, no. 05, May 2001.

[17] Regina Gumenyuk, Oleg G. Okhotnikov, "Impact of Gain Medium Dispersion on Stability of Soliton Bound States in Fiber Laser," IEEE Photonic Technology Letters, vol.25, no.02, Jan. 15,2013.

[18] Alaa Al-KAdry, Martin Rochelle, "Maximised Soliton Self-Frequency Shift in Non-Uniform Micro wires by the Control of Third Order Dispersion Perturbation," IEEE Journal of Lightwave Technology, vol.31, no. 09, May 2013.

[19] Shaofei Wang, Hairum Guo, Dengfeng Fan, "Analysis of Cascaded Soliton Spectral Tunneling Effect in Segmented Fibers with Engineered Dispersion," IEEE Photonics Journal, vol. 05, no. 06, Dec. 2013.

[20] Meng Liu, Ai-Ping Luo, Xu-Wu Zheng, Nian Zhao, Hao Liu, "Microfiber Based Highly Nonlinear Topological Insulator Photonic Device for the Formation of Versatile Multi-Soliton Patterns in a Fiber Laser," IEEE Journal of Lightwave Technology, vol.33, no. 10, May15,2015.

\section{BIOGRAPHIES}

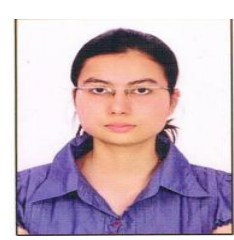

Suruchi Bala received the B.Tech degree in electronics and communication engineering from Rayat and Bahra College, Hoshiarpur, Punjab in 2014. She is currently pursuing Master degree in communication engineering at Guru Nanak Dev University. Her current research interests include the photonic technology and fiber communication.

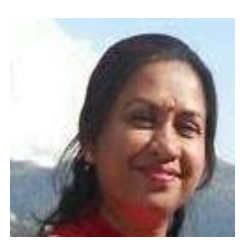

Anu Sheetal is Associate Professor at the Department of electronics and communication at Guru Nanak Dev University, RC Gurdaspur, Punjab. Her area of research interest is Digital Signal Processing and Optical Communication. 